\title{
Linear and non-linear price decentralization
}

\author{
Charalambos D. Aliprantis ${ }^{\mathrm{a}, *}$, Monique Florenzano $^{\mathrm{c}}$ \\ Rabee Tourky ${ }^{\mathrm{b}}$ \\ ${ }^{a}$ Department of Economics, Purdue University, West Lafayette, IN 47907-1310 \\ ${ }^{\mathrm{b}}$ Departments of Economics, The University of Melbourne, Melbourne, VIC 3010, \\ Australia; and Purdue University, West Lafayette, IN 47907-1310 \\ ${ }^{\mathrm{c}}$ CNRS-CERMSEM, Université Paris 1, 106-112 boulevard de l'Hôpital, 75647 \\ Paris Cedex 13, France
}

\begin{abstract}
Compendious and thorough solutions to the existence of a linear price equilibrium problem, the second welfare theorem, and the limit theorem on the core are provided for exchange economies whose consumption sets are the positive cone of arbitrary ordered Fréchet spaces - dispensing entirely with the assumption that the vector ordering of the commodity space is a lattice. The motivation comes from economic applications showing the need to bring within the scope of equilibrium theory vector orderings that are not lattices, which arise in the typical model of portfolio trading with missing options. The assumptions are on the primitives of the model and for $\omega$-proper economies they are both sufficient and necessary.
\end{abstract}

Key words: Linear and non-linear prices; equilibrium; welfare theorems Journal of Economic Literature Classification Numbers: C62, C71, D46, D51, D61.

* Corresponding author.

Email addresses: aliprantis@mgmt.purdue.edu (Charalambos D. Aliprantis), monique.florenzano@univ-paris1.fr (Monique Florenzano), rtourky@unimelb.edu.au (Rabee Tourky).

Preprint submitted to Elsevier Science

8 April 2004 


\section{Introduction}

The second half of the twentieth century saw an explosion of interest in the Walrasian model of general equilibrium. Of the many fundamental results that have been established three are foundational: the existence of at least one competitive equilibrium, the characterization of valuation equilibria as efficient allocations, and the characterization of competitive equilibria as Edgeworth equilibria.

These important results provide conceptual foundations for our understanding of efficiency and welfare economics. Moreover, all applied economic policy analysis relies implicitly on the foundations provided by general equilibrium theory. Unfortunately, at present general equilibrium theory does not provide an adequate understanding of economies in many settings. As a consequence, policy analysis in many important areas (e.g., financial markets) rests on shaky foundations.

For several decades a primary research program in general equilibrium theory has been to establish the classical theorems on price decentralization and the price equilibrium existence problem in a context sufficiently general to encompass as particular instances the important general equilibrium models which arise in applications. This program was well articulated by Mas-Colell [29]. The idea is to separate the very difficult mathematical problems associated with price decentralization from their applications.

The principal contributors to this program are, of course, Mas-Colell [29] and MasColell and Richard [30]. In those papers the authors replace the pervasive finite dimensional assumption on the interiority of endowments in consumption sets with two requirements:

(1) The commodity space be ordered by a vector lattice ordering that is "compatible" with the topology of the space and that constrains and motivates traders - the traders' consumption sets coincide with the positive cone of this ordering and preferences are monotone with respect to this ordering.

(2) That preferences satisfy an assumption termed $\omega$-uniform properness (where $\omega$ is the total endowment of resources), which is a cone condition that could in some settings be interpreted as a bound on the marginal rates of substitution (see for instance $[34,17,12,39]$ ).

The work of Mas-Colell [29] was extended in $[3,4,15,16,40,41]$ and the work of Mas- 
Colell and Richard [30] was extended in [20,22,32,33,37,38]. In all these papers some variant of $\omega$-properness is assumed and the lattice theoretic arguments are used in a non-trivial way. However, the use of lattice theoretic techniques comes at a price: the very structure of the economy is expressed in terms of the vector lattice ordering of the commodity space. For instance, the constraints on consumption sets, the notion of monotone preferences, the properness assumptions, free disposal, the notion of a free disposal equilibrium, the topology of the commodity space, and even the compactness assumptions on the set of feasible allocations are all defined with respect to the vector lattice ordering of the commodity space. Therefore, the economic meaning of these results hinges on the the interpretive efficacy of the ordering of the space.

Commodity spaces that are not lattice ordered arise naturally in many economic models and the large literature on price decentralization in vector lattices has little, that is obvious, to say in such a setting. An example of such an economic model is portfolio trading when markets are incomplete. It is known that in such models all the decentralization results can fail even if the preferences are uniformly proper and the commodity space is finite dimensional. In these models consumers are motivated by the payoff of a portfolio. Therefore, the meaningful natural ordering of the portfolio space is the one that compares portfolio payoffs and which is closely related to the notion of first order stochastic domination. In fact, the notion of arbitrage free prices is an order theoretic notion that induces this natural ordering of the portfolio space. Unfortunately, this ordering is rarely a vector lattice ordering when markets are not complete. The basic intuition for this is the following. Generally, when markets are not complete some call and put options cannot be replicated as the payoff of a portfolio of available securities. However, call and put options are closely related to the order structure of the portfolio space. Indeed, every marketed option is a lattice operation in the portfolio space and every lattice operation in the portfolio space is related to what is termed in the finance literature a minimum-cost super replicating portfolio of a call or put option (which need not exist).

Can we extend the existing results on price decentralization and the price equilibrium existence problem to ordered commodity spaces that are not vector lattices? We have the following four pointers:

(a) It is known that when the commodity space is an Archimedean ordered vector lattice the price decentralization results hold true but with possibly discontinuous prices. Jones [25] and Aliprantis and Burkinshaw [8] show that if the 
commodity space is a vector lattice, then to ensure that the decentralizing prices are continuous we need some lattice structure on the space of continuous prices.

(b) In the very general context where the commodity space is an ordered vector space that need not be a vector lattice, Aliprantis-Tourky-Yannelis [13] introduced an alternate value theory that arises from a personalized pricing system which induces a possibly non-linear value function. The classical price decentralization problems are solved by means of these non-linear value functions.

(c) Aliprantis-Monteiro-Tourky [9] give an example of a uniformly proper model of portfolio trading, with three securities and two traders (i.e., with a three dimensional commodity space), whose portfolio space is not lattice ordered and in which all the decentralization theorems fail for linear prices. In that example, optimal allocations can be supported by the Aliprantis-Tourky-Yannelis nonlinear prices.

(d) Aliprantis and Tourky [10] show that the dual cone of every ordered topological vector space can be embedded in a larger lattice ordered cone that contains nonlinear functionals, which they term the super order dual of the ordered vector space. Crucially, this embedding preserves lattice operations. By means of this embedding, they show that many of the useful structures of vector lattices have analogues in general ordered vector space settings. That is, ordered vector spaces are basically not so different from vector lattices.

Our purpose in this paper is to provide a thorough solution to the (linear) price decentralization and equilibrium existence problems in commodity spaces that are ordered Fréchet spaces, which includes the important class of ordered Banach spaces. The paper affords both necessary and sufficient conditions for linear price decentralization.

We deal with well behaved exchange economies that we call $\omega$-proper economies, which include the class of $\omega$-uniformly proper economies. We show that the linear decentralization of Pareto optimal allocations and Edgeworth equilibria as well as the existence of a linear price equilibrium are all basically equivalent to the properness of functionals in the super-order dual of the commodity space. More precisely, we consider the embedding of the dual cone of the commodity space in the in the super order dual, which is a lattice. Then we denote by $\mathcal{P}$ the semi-lattice generated by the cone of positive linear prices and call $\mathcal{P}$ our price space. Each possibly non-linear price $p$ in $\mathcal{P}$ can be written as the supremum $p=\bigvee_{i=1}^{m} f_{i}$, where $f_{1}, \ldots, f_{m}$ are positive linear prices. Now each function $p$ in $\mathcal{P}$ is a concave function defined on the 
positive cone of the commodity space. Therefore, the standard properness notions can be defined for these functions like any utility function. Surprisingly, it turns out that if each price in $\mathcal{P}$ satisfies a properness assumption, then the following hold true.

(1) The second welfare theorem holds, i.e., Pareto optimal allocations can be decentralized as (linear) Walrasian valuation equilibria.

(2) The limit theorem on the core holds, i.e., Edgeworth equilibria are (linear) Walrasian equilibria.

(3) With the standard compactness assumptions, there exists a (linear) Walrasian equilibrium.

The second major set of results of the paper shows that these same properness assumptions on the price space $\mathcal{P}$ are also necessary conditions for linear decentralization and the existence of equilibria.

The linear price decentralization theorems enumerated above afford positive results in a host of infinite dimensional settings that are not vector lattice ordered. We note that the $\omega$-properness of prices in $\mathcal{P}$ depends on the order structure of the commodity space and the "location" of the total endowment of resources. Furthermore, it is relatively easy to check if a price $p=\bigvee_{i=1}^{m} f_{i}$ satisfies our properness assumption. Indeed, in a large class of settings properness is equivalent to the existence of a solution to the following convex programming problem:

$$
\min g(\omega) \quad \text { subject to : } \quad g \text { is linear and } g \geq f_{1}, \ldots, f_{m} \text {. }
$$

In the finance framework this minimization problem translates into the following linear minimization problem:

$$
\min g(T(\omega)) \quad \text { subject to: } T^{\prime}(g) \geq f_{i} \text { for all } i=1, \ldots, m,
$$

where each $f_{i}$ is an arbitrage free securities price, $T$ is a portfolio returns operator that associates with each portfolio a state contingent claim, and $T^{\prime}$ is the adjoint of $T$ that associates with each Arrow-Debreu price a securities price. This second minimization problem is a variant of the canonical linear minimization problem and is the subject of much investigation in the mathematical literature on linear programming and operations research.

The main surprise of the analysis in the present paper is that the theory of value with linear prices can be articulated in terms of the cone conditions ubiquitously 
called properness in the economics literature. That is, the main contribution in the literature that follows the work of Mas-Colell [29] is the application of cone conditions to the primitive data of the economy rather than the application of lattice theoretic ideas to general equilibrium analysis.

The paper is organized as follows. Section 2 motivates the study in the paper by introducing an abstract framework of portfolio trading with incomplete markets. Our analysis begins in Section 3. The main results of the paper are contained in Sections 4 and 5. We return to financial models in the last section of the paper and apply our results.

\section{A Motivating Framework}

The purpose of this section is to introduce a very general securities model that includes as special cases many of the securities models studied in the literature. Our approach will be an operator theoretic study that is closely related to the setup of R. A. Jarrow, X. Jin, and D. B. Madan [24] and V. Galvani [23]. For a study of positive operators see the monographs $[1,7]$. We refer the reader to the next section of this paper for definitions concerning ordered vector spaces.

Let us summarize the basic framework. In a typical finance model one begins with a pair of function spaces $L$ and $X$, where $L$ is the portfolio space and $X$ is the space of all contingent claims, together with a portfolio returns operator $R: L \rightarrow X$ that satisfies the following two properties.

R1: There are no redundant securities (that is, $R$ is one-to-one) and $R$ is positive. R2: The operator $R$ pulls back order intervals of $R(L)$ to closed and bounded subsets of $L$.

The operator $R: L \rightarrow X$ reorders the portfolio space $L$ according to the ordering of $X$. This new ordering of the portfolio space is called the portfolio dominance ordering (a notion introduced in [5]), which in general is not a vector lattice ordering even if $\mathrm{X}$ is a vector lattice. Portfolio traders are motivated and constrained by this new ordering rather than the canonical ordering of $L$, which is basically redundant. Crucially, the arbitrage freeness of securities prices is relative to this new generally non-lattice portfolio dominance ordering. Some important examples of portfolio returns operators are provided by embeddings $R: L \hookrightarrow X$. That is, when $R(L)$ is 
closed or, equivalently, when $R$ is bounded below, i.e., there exists some constant $c>0$ such that $\|R \theta\| \geq c\|\theta\|$ holds for all $\theta \in L$; see [1, Theorem 2.5].

In a two period model the Banach lattice $X$ could be for instance one of the following:

(a) The Banach lattice $X=L_{2}(\mathcal{S}, \Sigma, \pi)$ of all square integrable functions on $\mathcal{S}$, where $(\mathcal{S}, \Sigma, \pi)$ is a probability space representing period one uncertainty.

(b) Given a compact topological space $\mathcal{S}$ representing the states of the world tomorrow, the Banach lattice $X=C(\mathcal{S})$ of all continuous functions on $\mathcal{S}$.

(c) Any of the Banach lattice commodity spaces from the existing literature.

In a continuous time model, we are given a probability space $(\mathcal{S}, \Sigma, \pi)$ and an interval $T \subseteq \mathbb{R}_{+}$such that the space $X$ is the space of all stochastic processes of the form $f(t, s)$, which are square integrable functions from $T$ to $L_{2}(\mathcal{S}, \Sigma, \pi)$.

Two examples in the finance literature of portfolio spaces are as follows.

(a) In a two-period model the available securities are indexed by a positive $\sigma$ finite measure space $(\mathcal{J}, \mathcal{F}, \mu)$. The portfolio space is the Banach space $L=$ $L_{2}(\mathcal{J}, \mathcal{F}, \mu)$. A portfolio is any vector in the portfolio space $L$.

(b) In a continuous time model where $T$ is a subinterval of $\mathbb{R}$, the space of portfolios is the space of portfolio processes from $T$ to $L_{2}(\mathcal{J}, \mathcal{F}, \mu)$. For more on portfolio processes we refer the reader to [26].

Two important examples of assets returns operators are presented next.

(1) When there are finitely many states of the world and finitely many available securities the assets returns operator $R$ is simply a non-negative matrix whose columns delineate the available non-redundant assets. For more on this example see for instance [28] and [35].

(2) In the case the portfolio space is the Banach function space $L=L_{2}(\mathcal{J}, \mathcal{F}, \mu)=$ $L_{2}(\mathcal{J})$ and the space of contingent claims is $X=L_{2}(\mathcal{S}, \Sigma, \pi)=L_{2}(\mathcal{S}), R$ is an integral operator. Here, the payoff of a security $j$ at state $s$ is denoted $P(j, s)$. The function $P: \mathcal{J} \times \mathcal{S} \rightarrow \mathbb{R}_{+}$is called the assets returns kernel, which is assumed to be $\mu \times \pi$-measurable. We assume that for each portfolio $\theta \in L$ the function $j \mapsto P(j, s) \theta(j)$ is $\mu$-integrable for $\pi$-almost all $s \in \mathcal{S}$. Moreover, the 
payoff $[R \theta](s)$ of a portfolio $\theta$ for $\pi$-almost all states of the world $s$ is given by

$$
[R \theta](s)=\int_{\mathcal{J}} P(j, s) \theta(j) d \mu(j)
$$

We assume that the range of the operator $R$ lies in $L_{2}(\mathcal{S})$. Therefore, $R$ defines an integral linear operator from the portfolio space $L_{2}(\mathcal{J})$ to the contingent claims space $L_{2}(\mathcal{S})$. In this case, notice that this operator is positive if and only if the assets returns kernel is non-negative.

In (2) above it is not always true that the portfolio returns operator $R$ pulls back order intervals of $R(L)$ to closed and bounded subsets of $L$. However, an example of a portfolio returns operator that pulls back order intervals of $R(L)$ to closed and bounded subsets of $L$ will be presented in Section 6 .

Let us formalize the general framework. We are given a Banach lattice $X$ that represents the space of all real (possibly multiperiod) contingent claims. A vector in $X$ is called a contingent claim. The positive cone of this space is $X_{+}$and, as usual, a consumer's consumption decision is restricted to this cone. Agents trade portfolios of available securities that pay contingent claims in $X$. They choose a portfolio which is a vector in a given Banach lattice $L$ called the portfolio space. The payoffs of portfolios are given by means of the portfolio returns operator $R: L \rightarrow X$. So, the payoff of a portfolio $\theta \in L$ is $R(\theta) \in X$ or simply $R \theta$.

A contingent claim $x \in X$ is said to be marketed if there exists a portfolio $\theta \in L$ such that $R \theta=x$. The vector subspace of all marketed contingent claims is denoted $\mathcal{M}$, and clearly, $\mathcal{M}=R(L)$, the range of $R$. We say that the market is complete if $\overline{\mathcal{M}}=X$, where $\overline{\mathcal{M}}$ denotes the closure of $\mathcal{M}$. If $\overline{\mathcal{M}} \neq X$, then the market is incomplete.

The portfolio returns operator induces a new ordering $\geq$ on the portfolio space $L$ as follows:

$$
\theta \geq \eta \Longleftrightarrow R \theta \geq R \eta
$$

This ordering is termed portfolio dominance. Denote the positive cone of this ordering of the portfolio space by $L_{+}=\{\theta \in L: R \theta \geq 0\}$, which is in general different from the canonical positive cone of the portfolio space $L$.

From now on when we talk about the ordering of the portfolio space we are referring to the portfolio dominance ordering. 
The portfolio space under this ordering fits perfectly into the analysis in the sequel.

Lemma 1 Under portfolio dominance the portfolio space is an ordered Banach space with a generating positive cone and bounded order intervals. If $L$ is reflexive or if $\mathcal{M}$ is closed and $X$ has an order continuous norm, then order intervals in the portfolio space are weakly compact.

proof. Notice first that the portfolio cone is generating since it contains the original positive cone of $L$, which is generating. Moreover, $L_{+}=R^{-1}\left(X_{+}\right)$is closed.

If $L$ is reflexive, then the dominance order intervals of $L$ are closed and bounded by assumption, and hence they are weakly compact. When $\mathcal{M}$ is closed, then by the open mapping theorem the operator $R: L \rightarrow \mathcal{M}$ is a topological and order isomorphism. Moreover, since $X$ has order continuous norm the order intervals of $X$ are weakly compact and so are the order intervals of $\mathcal{M}$. By the continuity of $R^{-1}: \mathcal{M} \rightarrow L$ it follows that the dominance order intervals of $L$ are likewise weakly compact.

Notice that commodity spaces that are Banach lattices with order continuous norms are ubiquitous in modern general equilibrium analysis.

Clearly, if $\mathcal{M}=X$, then portfolio dominance is a vector lattice ordering. This is so since the portfolio returns operator is surjective and therefore it reorders the portfolio space in a vector lattice manner. However, if the market is incomplete, then portfolio dominance is unlikely to be a vector lattice ordering. In fact, one can characterize the very rare situations in which this ordering is a vector lattice ordering.

For any set $Y$ in the space of contingent claims let $\mathcal{R}(Y)$ be the Riesz subspace of $X$ generated by $Y$, i.e., $\mathcal{R}(Y)$ is the smallest Riesz subspace containing $Y$.

Lemma 2 Portfolio dominance is a vector lattice ordering if and only if there exists a positive projection from $\mathcal{R}(\mathcal{M})$ to $\mathcal{R}(\mathcal{M})$ whose range is the marketed space $\mathcal{M}$.

proof. Clearly, portfolio dominance is a vector lattice ordering if and only if $\mathcal{M}$ is a Riesz space under its canonical ordering as a subspace of $X$. But by Miyajima's re- 
sult $\left[1\right.$, Theorem 5.64] this is the case if and only if there exists a positive projection ${ }^{1}$ from $\mathcal{R}(\mathcal{M})$ to $\mathcal{R}(\mathcal{M})$.

The lattice operations in the portfolio space are closely related to the presence of marketed call options. Recall, that a contingent claim $x \in X$ is called marketed if there exists a portfolio $\theta \in L$ satisfying $R \theta=x$. Now take a portfolio $\theta$ and a strike price $k \in X$. Assume that $k$ is marketed and let $\kappa \in L$ satisfy $R \kappa=k$. The call option on the portfolio $\theta$ with strike price $k$ is the contingent claim $[R \theta-k]^{+}=(R \theta-k) \vee 0 \in X$. The call option is non-trivial if neither $R \theta \geq k$ nor $R \theta \leq k$ hold. If such an option is marketed, then the supremum $[\theta-\kappa]^{+}=(\theta-\kappa) \vee 0$ exists in $L$ for the portfolio dominance ordering and $R[\theta-\kappa]^{+}=[R \theta-k]^{+}$.

In other words, a call option is a vector lattice operation in $X$ and if it is marketed, then it is replicated by a vector lattice operation in $L$. So, the marketability of call options transfers the lattice structure of $X$ to $L$. However, we know the following results from the finite dimensional setting.

Lemma 3 Suppose that there are J finitely many nominal securities and S finitely many states of the world. Let $\Phi$ be the space of all assets returns matrices for which the bond 1 is marketed. The following results hold true:

(1) Generically in $\Phi$ the market is complete if and only if all call options are marketed.

(2) If $J \leq \frac{1}{2}(S+1)$, then generically in $\Phi$ no non-trivial call option is marketed.

proof. For (1) see [35] and for (2) see [11].

Finally for this section, let us define the notion of arbitrage free securities prices. A securities price is simply a continuous linear functional on the portfolio space. Therefore, the dual space $L^{\prime}$ is called the space of securities prices. A securities price $p$ is weakly arbitrage free if for any portfolio $\theta \in L_{+}$(i.e., $R \theta \in X_{+}$) we have $p(\theta) \geq 0$. A securities price $p$ is arbitrage free if for any non-zero portfolio

1 An operator $T: V \rightarrow V$ on a vector space is called a projection if $T^{2}=T$. If $V$ is an ordered vector space a projection that is also a positive operator is called a positive projection. 
$\theta \in L_{+} \backslash\{0\}$ we have $p(\theta)>0$. Therefore, arbitrage freeness is defined in terms of the portfolio dominance ordering of $L$.

In fact, portfolio dominance induces an ordering on the space of securities prices as follows: $p \geq q$ if $p(\theta) \geq q(\theta)$ for all $\theta \in L_{+}$. This ordering is a vector ordering that makes $L^{\prime}$ an ordered Banach space. As usual denote by $L_{+}^{\prime}$ the positive cone of $L^{\prime}$. That is, $L_{+}^{\prime}=\left\{f \in L^{\prime}: f \geq 0\right\}$.

Lemma 4 A securities price $p$ is weakly arbitrage free if and only if $p \in L_{+}^{\prime}$. It is arbitrage free if and only if it is a strictly positive linear functional (i.e., $p \gg 0$ ).

\section{The General Equilibrium Model}

In this section we introduce the commodity space, the price space, proper economies, and the various notions of equilibria. For technical details not covered in this paper, we refer the reader to the following monographs. For general infinite dimensional analysis see [2]. For the theory of ordered topological vector spaces see [31,36]. For vector lattices see $[6,27]$. We shall extensively use results obtained in [10] regarding the super order dual of an ordered vector space.

The Commodity Space is an ordered Fréchet space $L$ with a closed and generating positive cone whose order intervals are topologically bounded. That is:

(a) $L$ is a topological vector space endowed with a complete metrizable locally convex topology $\tau$.

(b) $L$ is equipped with an order relation $\geq$ such that $x \geq y$ imply $\alpha x+z \geq \alpha y+z$ for all $z \in L$ and all scalars $\alpha \geq 0$.

(c) The positive cone $L_{+}=\{x \in L: x \geq 0\}$ is closed and generating, i.e., $L_{+}-L_{+}=L$.

(d) Each order interval $[0, x]=\left\{y \in L_{+}: 0 \leq y \leq x\right\}$ is $\tau$-bounded.

The topological dual of $(L, \tau)$ is denoted $L^{\prime}$. It is also an ordered vector space under its canonical ordering, i.e., $f \geq g$ if $f(x) \geq g(x)$ for all $x \in L_{+}$. We shall require that at least one of Mas-Colell's [29] quasi-concave uniformly proper functions that is also strictly monotone exists, or equivalently: 
- There exists a strictly positive linear functional $f \in L^{\prime}$, i.e., $f(x)>0$ for all $x>0$.

The dual of our commodity space is well behaved.

Lemma 5 Every positive linear functional is continuous and the cone $L_{+}^{\prime}$ is generating. ${ }^{2}$

If the least upper bound (supremum) (resp. the greatest lower bound (infimum)) of a subset $S$ of an ordered space exists, then it may be denoted $\sup S$ or $\bigvee_{i=1}^{m} x_{i}$ when $S=\left\{x_{1}, \ldots, x_{m}\right\}$ or $x \vee y$ when $S=\{x, y\}$ (resp. inf $S$ or $\bigwedge_{i=1}^{m} x_{i}$ or $x \wedge y$ ). As usual, we shall say that $L$ is a Riesz space (or a vector lattice) if $x \vee y$ and $x \wedge y$ exist in $L$ for all $x, y \in L$.

The Price Space will be a semi-lattice of the super order dual of $L_{+}$. We begin with a few definitions. We say that a function $f: L_{+} \rightarrow \mathbb{R}$ is positive if $f(x) \geq 0$ holds for each $x \in L_{+}$; is super-additive, if $f(x)+f(y) \leq f(x+y)$ holds for all $x, y \in L_{+}$; additive if $f(x+y)=f(x)+f(y)$ holds for all $x, y \in L_{+}$; and is positively homogeneous, if $f(\alpha x)=\alpha f(x)$ holds for every $\alpha \geq 0$ and all $x \in L_{+}$.

The price space will be a subset of the following collection of real-valued non-negative functions on $L_{+}$given by

$$
\Pi=\left\{f \in \mathbb{R}_{+}^{L_{+}}: \quad f \text { is super-additive and positively homogeneous }\right\} .
$$

It should be noticed that every function $f \in \Pi$ is monotone in the sense that $0 \leq x \leq y$ implies $0=f(0) \leq f(x) \leq f(y)$. Clearly, $\Pi$ is a cone of $\mathbb{R}^{L_{+}}$. The cone $\Pi$ is called the super order dual cone of $L_{+}$and was introduced and studied in detail in [10]. Notice that $\Pi$ inherits the natural pointwise order of $\mathbb{R}^{L_{+}}$. In other words, for $f, g: L_{+} \rightarrow \mathbb{R}$ we write $f \geq g$ (and say that $f$ dominates $g$ ) whenever $f(x) \geq g(x)$ holds for each $x \in L_{+}$.

The important result for us here is that the cone $\Pi$ is a lattice even though $L$ may

$\overline{2}$ From [10, Theorem 7.2] we know that $L^{\prime}=L^{r}=L^{\sim}$ where $L^{r}$ is the order regular dual of $L$ comprising of linear functionals that can be written as differences of positive linear functionals and $L^{\sim}$ is the order dual of $L$ consisting of all order bounded linear functionals on $L$. 
not be a vector lattice; see [10, Corollary 4.4].

Theorem 6 The super order dual cone $\Pi$ with its natural ordering is a lattice. Moreover, the supremum operation for any pair $f_{1}, f_{2} \in \Pi$ and any $x \in L_{+}$is given by

$$
\left[f_{1} \vee f_{2}\right](x)=\sup \left\{f_{1}\left(y_{1}\right)+f_{2}\left(y_{2}\right): y_{1}, y_{2} \in L_{+} \text {and } y_{1}+y_{2}=x\right\}
$$

The dual cone $L_{+}^{\prime}$ can be viewed as a subcone of the cone $\Pi$. In particular, throughout our discussion we shall use the following simple but very useful result, whose proof follows from the Kantorovich extension theorem; see for instance [1, Theorem 1.15].

Lemma 7 The positive linear functionals on $L$ are precisely the extensions of the additive functions in $\Pi$. So, we can write $L_{+}^{\prime}=\{f \in \Pi: \quad f$ is additive $\}$.

As a matter of fact, there is a natural embedding $J: L_{+}^{\prime} \rightarrow \Pi$ defined by

$$
J(f)(x)=f(x)
$$

for all $x \in L_{+}$. This embedding is clearly one-to-one and monotone. In addition, we have the following remarkable result proved in [10, Theorem 5.5].

Theorem 8 (Aliprantis-Tourky) Assume that the order intervals of L are weakly compact. If the supremum $\bigvee_{i=1}^{n} f_{i}$ of the positive continuous linear functionals $f_{1}, \ldots, f_{n}$ on $L$ exists in $L^{\prime}$, then

$$
J\left(\bigvee_{i=1}^{n} f_{i}\right)=\bigvee_{i=1}^{n} J\left(f_{i}\right)
$$

holds in $\Pi$. That is, the embedding $J: L_{+}^{\prime} \rightarrow \Pi$ preserves finite suprema.

Let us say that a non-empty subset of a lattice is a semi-lattice if it is closed under finite suprema. Every non-empty subset $A$ of a lattice is included in a smallest semilattice $\mathcal{U}(A)$, called the semi-lattice generated by $A$. Clearly, $\mathcal{U}(A)$ consists of all finite suprema of the set $A$. With this definition in mind, we are now ready to define our price space.

Definition 9 The price space $\mathcal{P}$ is the semi-lattice $\mathcal{U}\left(L_{+}^{\prime}\right)$, i.e., $\mathcal{P}$ consists of all finite suprema of $L_{+}^{\prime}$ in the super dual cone $\Pi$.

The pair $\langle L, \mathcal{P}\rangle$ will be our commodity-price duality. In the special case of a Riesz commodity space, i.e., when $L$ is a Fréchet lattice, the price space $\mathcal{P}$ coincides 
with the positive cone of $L^{\prime}$ and we regain the standard commodity-price duality in the literature. We have the following result concerning the price space $\mathcal{P}$.

Lemma 10 If $\mathcal{P}=L_{+}^{\prime}$, then $L^{\prime}$ is a Riesz space. Conversely, $\mathcal{P}=L_{+}^{\prime}$ whenever one of the following conditions holds true:

(1) L is a Riesz space.

(2) L has the Riesz decomposition property, i.e., $[0, x]+[0, y]=[0, x+y]$ holds for all $x, y \in L_{+}$.

(3) $L$ is a Banach space and $L^{\prime}$ is a Riesz space in its own right.

(4) The order intervals of $L$ are weakly compact and $L^{\prime}$ is a Riesz space in its own right. ${ }^{3}$

proof. First suppose that $\mathcal{P}=L_{+}^{\prime}$. Fix $f \in L^{\prime}$. Since $L^{\prime}=L^{r}$ holds, it follows that there exists some $g \in L_{+}^{\prime}$ satisfying $-g \leq f$. By our assumption $(f+g) \vee g=g+f \vee 0$ exits in $\mathcal{P}=L_{+}^{\prime}$. This implies that $f \vee 0$ exists in $L^{\prime}$. Therefore $L^{\prime}$ is a Riesz space.

The proofs of (1)-(4) follow from the fact that in those settings for any $f_{1}, f_{2} \in L_{+}^{\prime}$ and for any $x \in L_{+}$the supremum is given by the Riesz-Kantorovich formula:

$$
\left[f_{1} \vee f_{2}\right](x)=\sup \left\{f_{1}\left(y_{1}\right)+f_{2}\left(y_{2}\right): y_{1}, y_{2} \in L_{+} \text {and } y_{1}+y_{2}=x\right\}
$$

in both $L^{\prime}$ and in $\Pi$. See [14] for (3) and [10] for (4).

Each price in $\mathcal{P}$ can be written as the supremum $\bigvee_{i=1}^{n} f_{i}$ of linear functions $f_{1}, \ldots, f_{n}$ in $L_{+}^{\prime}$. So, from now on, $\bigvee_{i=1}^{n} f_{i}$ shall be understood to be the price in $\mathcal{P}$ which is the supremum of the linear functions $f_{1}, \ldots, f_{n} \in L_{+}^{\prime}$.

Perhaps we can appreciate why in general the suprema in $\mathcal{P}$ are not additive by looking at the special case of a three dimensional commodity space. The half-ray of the cone $L_{+}$determined by a vector $x \in L_{+}$is the set $\mathcal{L}_{x}=\{\alpha x: \alpha \geq 0\}$. A

3 We note that every Riesz space has the Riesz decomposition property, but not every space that has the Riesz decomposition property is a Riesz space. The following is a open question in the mathematical literature: Does there exist an ordered vector space that does not have the Riesz decomposition property and whose regular dual $L^{r}$ is a vector lattice? 
half-ray $\mathcal{L}_{x}$ of $L_{+}$is called an extremal ray of the cone $L_{+}$if for each $y \in \mathcal{L}_{x}$ we have $[0, y]=\{\delta y: 0 \leq \delta \leq 1\}$. The following result is easy to prove; see [10].

Lemma 11 Let $L$ be a three dimensional vector space. There exist at least one pair of non-comparable linear functionals $f, g \in L_{+}^{\prime}$ such that the function $f \vee g \in \mathcal{P}$ is additive if and only if $L_{+}$has at most four extremal rays.

Domination in $\mathcal{P}$ is characterized as follows; for a proof see [10, Lemma 3.7].

Lemma 12 (Aliprantis-Tourky) Assume that $f, g \in \mathcal{P}$. If $f\left(x_{0}\right)>0$ holds for some $x_{0} \in L_{+}$, then the following statements are equivalent.

(1) The function $g$ dominates $f$, i.e., $g(y) \geq f(y)$ holds for each $y \in L_{+}$.

(2) For each $y \in L_{+}$satisfying $f(y)=f\left(x_{0}\right)$ we have $g(y) \geq f\left(x_{0}\right)$.

The Economy has $m$ consumers designated by the index $i=1, \ldots, m$. As usual, the consumption set of consumer $i$ is $L_{+}$. The bundle $\omega_{i}>0$ is the $i$ th consumer's initial endowment and $\omega=\sum_{i=1}^{m} \omega_{i}$ is the total endowment.

The correspondence $P_{i}: L_{+} \rightarrow L_{+}$denotes the $i$ th consumer's (strict) preferences. We assume that each mapping $P_{i}$ satisfies the standard assumptions. That is, $P_{i}$ is irreflexive, i.e., $x \notin P_{i}(x)$ for each $x \in L_{+}$; is strictly monotone, i.e., $x+y \in$ $P_{i}(x)$ for each $y>0$; is convex-valued; has open values in $L_{+}$for some linear topology on $L$; and is $\omega$-proper in the sense of Tourky [37]:

- There exists another convex-valued correspondence $\widehat{P}_{i}: L_{+} \rightarrow L$ such that for each $x \in L_{+}$:

(a) The vector $x+\omega$ is an interior point of $\widehat{P}_{i}(x)$.

(b) $\widehat{P}_{i}(x) \cap L_{+}=P_{i}(x)$.

An exchange economy with commodity-price duality $\langle L, \mathcal{P}\rangle$ and with the above characteristics will be referred to as a proper economy. We will say that the economy is compact if order intervals of $L$ are weakly compact and preferences have weakly open lower sections, that is, for each bundle $y \in L_{+}$the lower section $P_{i}^{-1}(y)=\left\{x \in L_{+}: y \in P_{i}(x)\right\}$ is weakly open in $L_{+}$.

For any $x \in L_{+}$let $\mathcal{A}_{x}=\left\{y \in L_{+}^{m}: \sum_{i=1}^{m} y_{i}=x\right\}$. As usually, $\left(x_{1}, \ldots, x_{m}\right) \in \mathcal{A}_{\omega}$ is said to be an allocation. In our general setting, we say that endowments are 
decomposable if

$$
A_{\omega_{1}}+A_{\omega_{2}}+\cdots+A_{\omega_{m}}=\mathcal{A}_{\omega} .
$$

This is a very natural assumption in economics. It means that each allocation can be viewed as the outcome of bilateral trading. Notice that in the presence of the Riesz decomposition property this condition is always true. However, this equality can be true for some choices of initial endowments in any ordered vector space.

Definition 13 For a given commodity space $L$ and any fixed $\omega>0$, we shall denote the collection of all proper economies with commodity-price duality $\langle L, \mathcal{P}\rangle$ and total endowment $\omega$ by $\mathcal{E}(L, \omega)$. The collection of economies in $\mathcal{E}(L, \omega)$ for which the endowments are decomposable will be denoted $\mathcal{E}_{D}(L, \omega)$.

The next result informs us that we are not working with an empty set of economies.

Lemma 14 For any commodity space $L$ and any fixed $\omega>0$ the collection of all decomposable economies $\mathcal{E}_{D}(L, \omega) \subseteq \mathcal{E}(L, \omega)$ is non-empty and contains economies with arbitrarily many consumers. Further, if L has the Riesz decomposition property, then $\mathcal{E}(L, \omega)=\mathcal{E}_{D}(L, \omega)$.

proof. To see that $\mathcal{E}_{D}(L, \omega)$ is non-empty, let $\omega_{i}=\frac{1}{m} \omega$ and assume that every consumer $i$ has the utility function $u_{i}(x)=f(x)$, where $f \in L^{\prime}$ is a strictly positive linear functional.

Let us introduce the standard notions of efficiency. An allocation $\left(x_{1}, \ldots, x_{m}\right)$ is said to be weakly Pareto optimal, if there is no allocation $\left(y_{1}, \ldots, y_{m}\right)$ satisfying $y_{i} \in P\left(x_{i}\right)$ for each $i \in I$; a core allocation, if it cannot be blocked by any allocation in the sense that there is no allocation $\left(y_{1}, \ldots, y_{m}\right)$ and a coalition $S$ such that

(a) $\sum_{i \in S} y_{i}=\sum_{i \in S} \omega_{i}$, and

(b) $y_{i} \in P_{i}\left(x_{i}\right)$ for all $i \in S$;

an Edgeworth equilibrium, if it belongs to the core of every $r$-fold replica economy. ${ }^{4}$

$\overline{4}$ The notion of Edgeworth equilibrium was introduced in [3] and was inspired by the notion of the strict core of G. Debreu and H. E. Scarf [19]. It was shown in [21] that 
A Price Equilibrium in this paper is the generalization in [13] of the standard notion of Walrasian equilibrium to the case of the extended commodity price duality $\langle L, \mathcal{P}\rangle$.

Definition 15 An allocation $\left(x_{1}, \ldots, x_{m}\right)$ is said to be:

(1) A valuation equilibrium, if there exists some price $p \in \mathcal{P}$ (called a supporting price) such that:

(a) $y \in P_{i}\left(x_{i}\right)$ implies $p(y)>p\left(x_{i}\right)$, and

(b) the arbitrage-free condition $0<p(\omega) \leq \sum_{i=1}^{m} p\left(x_{i}\right)$ holds.

(2) An equilibrium, if there exists some some price $p \in \mathcal{P}$ (again called a supporting price) such that:

(a) $p(\omega)>0$,

(b) $y \in P_{i}\left(x_{i}\right)$ implies $p(y)>p\left(x_{i}\right)$, and

(c) for each $\left(\alpha_{1}, \ldots, \alpha_{m}\right) \in \mathbb{R}_{+}^{m}$ we have $p\left(\sum_{i=1}^{m} \alpha_{i} \omega_{i}\right) \leq \sum_{i=1}^{m} \alpha_{i} p\left(x_{i}\right)$.

(3) A personalized equilibrium (resp. a personalized valuation equilibrium), whenever it is an equilibrium (resp. a valuation equilibrium) with respect to some supporting price of the form $p=\bigvee_{i=1}^{m} f_{i}$ with $f_{i} \in L_{+}^{\prime}$ for each $i$ that satisfies $p(\omega)=\sum_{i=1}^{m} f_{i}\left(x_{i}\right)$.

(4) A Walrasian equilibrium (resp. a Walrasian valuation equilibrium), if it is an equilibrium (resp. a valuation equilibrium) with an additive supporting price in $\mathcal{P}$.

Notice that condition (b) of part (1) of the above definition is equivalent to:

$$
p(\omega)=\sum_{i=1}^{m} p\left(x_{i}\right)>0 .
$$

In view of Lemma 7 it is easy to see that our definition of a Walrasian equilibrium coincides with the standard definition in the literature. The following theorem, established in [13, Theorems 5.1 and 7.5 \& Lemma 6.3], is the basic result in the literature regarding the decentralization of efficient allocations by non-linear prices.

Theorem 16 (Aliprantis-Tourky-Yannelis) For a proper economy the following hold true:

(1) An allocation is weakly Pareto optimal if and only if it is a valuation equilibrium.

Edgeworth equilibria exist in compact economies. 
(2) An allocation is an Edgeworth equilibrium if and only if it is an equilibrium.

(3) Every equilibrium is a personalized equilibrium and every valuation equilibrium is a personalized valuation equilibrium.

(4) If the economy is compact, then there exists an equilibrium.

We also have the following companion to Theorem 16 that was obtained in [9].

Theorem 17 There exists a compact proper economy in which an equilibrium with a non-linear price exists but no Walrasian equilibrium with (continuous or discontinuous) linear prices exists, and Pareto optimal allocations that are supported as equilibria by non-linear prices but not as Walrasian valuation equilibria with linear prices.

\section{Linear Decentralization}

In this section we look at sufficient conditions on the order structure of the space that will guarantee the supportability of optimal allocations by linear prices. But first, let us recall some notions of properness for functions on $L_{+}$.

Definition 18 We say that a function $f: L_{+} \rightarrow \mathbb{R}$ is:

(1) $\omega$-proper at some $y \in L_{+}$, if there exists a convex set $F$ such that:

(a) $y+\omega$ is an interior point of $F$, and

(b) $F \cap L_{+}=\left\{z \in L_{+}: f(z)>f(y)\right\}$.

(2) Pointwise proper at $\omega$, if there exists an open wedge ${ }^{5} \Gamma$ such that:

(a) $(\omega-\Gamma) \cap L_{+} \neq \varnothing$, and

(b) $(\omega-\Gamma) \cap\left\{x \in L_{+}: f(x)>f(\omega)\right\}=\varnothing$.

Some basic properties of the preceding notions of properness are included in the next result.

Lemma 19 For a function $f: L_{+} \rightarrow \mathbb{R}$ we have the following.

(1) If there exists a price $\pi \in L_{+}^{\prime}$ that supports the set $\left\{z \in L_{+}: f(z)>f(\omega)\right\}$ at $\omega$ (i.e., $z \in L_{+}$with $f(z)>f(\omega)$ implies $\pi(z) \geq \pi(\omega)$ ) and satisfies $\pi(\omega)>0$,

$\overline{5}$ An open wedge $\Gamma$ is any nonempty open convex set such that $\alpha x \in \Gamma$ for any $\alpha>0$ and $x \in \Gamma$. 
then $f$ is pointwise proper at $\omega$.

(2) A price $p \in \mathcal{P}$ that satisfies $p(\omega)>0$ is pointwise proper at $\omega$ if and only if there exists some price $\pi \in L_{+}^{\prime}$ such that:

(a) $\pi(\omega)=p(\omega)$,

(b) $\pi \geq p$, and

(c) $\pi$ supports the convex set $\left\{z \in L_{+}: p(z) \geq p(\omega)\right\}$ at $\omega$.

(3) If $f$ is $\omega$-proper at $\omega$, then $f$ is pointwise proper at $\omega$.

proof. (1) Assume that the set $\left\{z \in L_{+}: f(z)>f(\omega)\right\}$ is supported at $\omega$ by a price $\pi \in L_{+}^{\prime}$ that satisfies $\pi(\omega)>0$. Now consider the open wedge $\Gamma=\{x \in L: \pi(x)>$ $0\}$ and note that $\omega-\frac{1}{2} \omega \in(\omega-\Gamma) \cap L_{+}$and that if some $\gamma \in \Gamma$ satisfies $\omega-\gamma \in L_{+}$ and $f(\omega-\gamma)>f(\omega)$, then $\pi(\omega) \leq \pi(\omega-\gamma)$ or $\pi(\gamma) \leq 0$, which is impossible. Hence, $(\omega-\Gamma) \cap\left\{x \in L_{+}: f(x)>f(\omega)\right\}=\varnothing$, and thus $f$ is pointwise proper at $\omega$.

(2) Assume that $\Gamma$ is an open wedge such that $(\omega-\Gamma) \cap\left\{z \in L_{+}: p(z)>p(\omega)\right\}=\varnothing$ and $(\omega-\Gamma) \cap L_{+} \neq \varnothing$. By the separation theorem there exists some non-zero $\pi \in L^{\prime}$ such that $\pi(\omega-\gamma) \leq \pi(z)$ holds for all $\gamma \in \Gamma$ and all $z \in L_{+}$with $p(z)>p(\omega)$. From $p(\delta \omega)>p(\omega)$ for all $\delta>1$ we get $\pi(\omega-\gamma) \leq \delta \pi(\omega)$ for all $\delta>1$. This implies $\pi(\gamma) \geq 0$ for all $\gamma \in \Gamma$, and consequently $\pi(\gamma)>0$ for all $\gamma \in \Gamma .{ }^{6}$

To see that $\pi \geq 0$ holds fix $z \in L_{+}$and note that for each real number $\delta>1$ we have $p(\delta \omega+z)>p(\omega)$. This implies $\pi(\omega-\gamma) \leq \delta \pi(\omega)+\pi(z)$ for all $\delta>1$ and all $\gamma \in \Gamma$, and from this it follows that $\pi(z) \geq 0$, i.e., $\pi \geq 0$. Next, pick some $\gamma_{1} \in \Gamma$ with $\omega-\gamma_{1} \in L_{+}$and note that $0 \leq \pi\left(\omega-\gamma_{1}\right)<\pi(\omega)$. Moreover, since $p$ is concave, it should be clear that $\pi$ supports the set $\left\{z \in L_{+}: p(z) \geq p(\omega)\right\}$ at $\omega$. If we replace $\pi$ by $\frac{p(\omega)}{\pi(\omega)} \pi$ we have $\pi(\omega)=p(\omega)$. The fact that $\pi \geq p$ follows immediately from Lemma 12. The converse follows immediately from part (1).

(3) Assume that $f$ is $\omega$-proper at $\omega$. Pick a convex set $F$ such that $2 \omega$ in an interior point of $F$ and $F \cap L_{+}=\left\{z \in L_{+}: f(z)>f(\omega)\right\}$. Since $\omega \notin\left\{z \in L_{+}: f(z)>f(\omega)\right\}$, it follows that $\omega \notin F$. So, by the separation theorem, there exists some non-zero $\pi \in L^{\prime}$ such that $\pi(\omega) \leq \pi(x)$ for all $x \in F$. Since $2 \omega$ is an interior point of $F$, it follows that $\pi(\omega)>0$. Now notice that $\pi$ supports the set $\left\{z \in L_{+}: f(z)>f(\omega)\right\}$

6 If $\pi\left(\gamma_{0}\right)=0$ holds for some $\gamma_{0} \in \Gamma$, then pick some circled neighborhood $V$ of zero with $\gamma_{0}+V \subseteq \Gamma$ and note that $\pi\left(\gamma_{0}+v\right) \geq 0$ implies $\pi(v) \geq 0$ for all $v \in V$. The latter shows that $\pi=0$, which is a contradiction. 
at $\omega$ and use part (1).

We also have the following useful characterization of pointwise proper prices.

Lemma 20 If order intervals of $L$ are weakly compact, then for a price $p$ of the form $p=\bigvee_{i=1}^{m} f_{i}$ such that $p(\omega)>0$ the following statements are equivalent:

(1) The price $p$ is pointwise proper at $\omega$.

(2) There exists a solution to the following linear minimization problem:

$$
\min g(\omega) \text { subject to: } g \in L^{\prime} \text { and } g \geq f_{i} \text { for all } i=1, \ldots, m \text {. }
$$

proof. (1) $\Longrightarrow(2)$ If $p=\bigvee_{i=1}^{m} f_{i}$ is pointwise proper at $\omega$, then (according to part (2) of Lemma 19) there is some $\pi \in L_{+}^{\prime}$ with $\pi \geq p$ and $\pi(\omega)=p(\omega)$. Clearly, $\pi \geq f_{i}$ for all $i$ and $\pi$ is a solution to the minimization problem $(\mathcal{S})$.

$(2) \Longrightarrow(1)$ Let $p=\bigvee_{i=1}^{m} f_{i}$. Let $\pi$ be a solution to the minimization problem $(\mathcal{S})$. From [10, Theorem 7.6] it follows that $p(\omega)=\pi(\omega)>0$. From the fact that $\pi \geq f_{i}$ for each $i$ we see that $\pi \geq p$ and (by Lemma 12) $\pi$ supports the convex set $\{y \in$ $\left.L_{+}: p(y)>p(\omega)\right\}$ at $\omega$. By part (1) of Lemma 19, $p$ is pointwise proper at $\omega$.

In the next result we show how pointwise properness of non-linear prices can guarantee decentralization by linear prices.

Lemma 21 We have the following:

(1) A valuation equilibrium supported by a price that is pointwise proper at $\omega$ is a Walrasian valuation equilibrium.

(2) An equilibrium supported by a price that is $\omega$-proper at $\omega$ is a Walrasian equilibrium.

(3) If the initial endowments are decomposable, then any equilibrium supported by a price that is pointwise proper at $\omega$ is a Walrasian equilibrium.

proof. (1) Let $\left(x_{1}, \ldots, x_{m}\right)$ be a valuation equilibrium that is supported by a price $p \in \mathcal{P}$ which is pointwise proper at $\omega$. We can assume that $p(\omega)=1$. By part (2) of 
Lemma 19 there exists some $\pi \in L_{+}^{\prime}$ satisfying $\pi(\omega)=p(\omega)=1$ that supports the set $\left\{z \in L_{+}^{\prime}: p(z) \geq p(\omega)\right\}$ at $\omega$ and $\pi \geq p$. Now the relation

$$
1=\pi(\omega)=\sum_{i=1}^{m} \pi\left(x_{i}\right) \geq \sum_{i=1}^{m} p\left(x_{i}\right)=p(\omega)=1
$$

implies that $\pi\left(x_{i}\right)=p\left(x_{i}\right)$ for each $i$. It is now easy to see that $\pi$ supports the allocation as a Walrasian valuation equilibrium.

(2) Let $\left(x_{1}, \ldots, x_{m}\right)$ be an equilibrium with a supporting price $p \in \mathcal{P}$ satisfying $p(\omega)=1$. Since preferences are strictly monotone and $\alpha x_{i} \in P_{i}\left(x_{i}\right)$ holds for each $\alpha>1$ it follows that $p\left(\alpha x_{i}\right)=\alpha p\left(x_{i}\right)>p\left(x_{i}\right)$. This implies that $p\left(x_{i}\right)>0$ for each $i$.

Now consider the collection of bundles

$$
X=\left\{\frac{\omega_{1}}{p\left(x_{1}\right)}, \ldots, \frac{\omega_{m}}{p\left(x_{m}\right)}\right\}
$$

Since $\sum_{i=1}^{m} p\left(x_{i}\right)=p(\omega)=1$, it is easy to see that $\omega \in \operatorname{co} X$. Also, from condition (c) of the definition of an equilibrium, it follows that

$$
\operatorname{co} X \cap\left\{z \in L_{+}: p(z)>1\right\}=\varnothing
$$

From the $\omega$-properness of $p$ we see that there exists some convex set $F$ that contains $2 \omega$ in its interior such that $F \cap L_{+}=\left\{z \in L_{+}: p(z)>1\right\}$. Therefore, co $X \cap F=\varnothing$. Let $\pi$ be a non-zero continuous linear functional that separates these two sets, i.e., $\pi(x) \leq \pi(z)$ holds for all $x \in \operatorname{co} X$ and all $z \in F$ with $p(z)>1$. Since $\omega \in \operatorname{co} X$ and $2 \omega$ is in the interior of $F$, we see that that $\pi(2 \omega)>\pi(\omega)$, which implies that $\pi(\omega)>0$. Therefore, we can assume that $\pi(\omega)=1$.

Now if $z \in L_{+}$satisfies $p(z)=p(\omega)=1$, then $\delta z \in F$ for all $\delta>1$ and therefore we have $p(\omega)=\pi(\omega) \leq \delta \pi(z)$ for all $\delta>1$. This implies $p(\omega) \leq \pi(z)$, and consequently from Lemma 12 it follows that $\pi \geq p$. Therefore, as in part (1), it must be the case that $\pi$ supports the allocation as a Walrasian valuation equilibrium with $\pi\left(x_{i}\right)=$ $p\left(x_{i}\right)$ for each $i$. Furthermore, it should be clear that $\pi\left(\frac{\omega_{i}}{p\left(x_{i}\right)}\right) \leq 1$ and therefore, $\pi\left(\omega_{i}\right) \leq p\left(x_{i}\right)=\pi\left(x_{i}\right)$. This implies $\pi\left(\omega_{i}\right)=\pi\left(x_{i}\right)$ and thus $\pi$ supports the allocation as a Walrasian equilibrium. 
(3) Assume that $\sum_{i=1}^{m} \mathcal{A}_{\omega_{i}}=\mathcal{A}_{\omega}$ and let $\left(x_{1}, \ldots, x_{m}\right)$ be an equilibrium. By part (3) of Theorem 16 we know that $\left(x_{1}, \ldots, x_{m}\right)$ is a personalized equilibrium. Let $p=$ $\bigvee_{i=1}^{m} f_{i}$ be a personalized price supporting $\left(x_{1}, \ldots, x_{m}\right)$ with $p(\omega)=1$. We know that

$$
p(\omega)=\sup \left\{\sum_{i=1}^{m} f_{i}(y): y \in A_{\omega}\right\} .
$$

Now from the decomposability assumption, it follows that $\sum_{i=1}^{m} p\left(\omega_{i}\right)=p(\omega)$. In particular, $p\left(x_{i}\right)=p\left(\omega_{i}\right)$ for each $i$. Now as in (1) we can find some $\pi \in L_{+}^{\prime}$ with $\pi(\omega)=1$ and $\pi \geq p$. As above $\pi\left(x_{i}\right)=p\left(x_{i}\right)$ and $\pi\left(\omega_{i}\right)=p\left(\omega_{i}\right)$, which clearly implies that $\pi$ is the desired supporting linear price.

We continue by introducing the notion of a proper price space.

Definition 22 We shall say that the price space $\mathcal{P}$ is:

(1) $\omega$-proper, if each price $p \in \mathcal{P}$ satisfying $p(\omega)>0$ is $\omega$-proper at $\omega$, and

(2) pointwise proper at $\omega$, if each price $p \in \mathcal{P}$ satisfying $p(\omega)>0$ is pointwise proper at $\omega$.

From Lemma 19 we know that an $\omega$-proper price space $\mathcal{P}$ is automatically pointwise proper at $\omega$. We can now turn to the first of our major results. Remarkably, as expected from the previous discussion, when the price space is proper we regain the classical Walrasian value theory with linear prices even outside of the realm of vector lattice commodity spaces.

Theorem 23 For an w-proper price space we have the following:

(1) The notions of equilibrium, Walrasian equilibrium, and Edgeworth equilibrium coincide.

(2) If the economy is compact, then there exists a Walrasian equilibrium.

proof. The validity of the statements follows immediately from Lemma 21 in conjunction with Theorem 16.

There are many interesting examples of price spaces that are $\omega$-proper for which the commodity space is not a Riesz space and lacks the Riesz decomposition property. 
Moreover, the properness of the price space depends on the position of $\omega$ in $L_{+}$. One can construct even finite dimensional examples in which the price space is proper for some positive vectors but not for others.

We conclude this section with an analogue of the preceding theorem for pointwise proper price spaces. Its proof is similar and is omitted.

Theorem 24 For a price space that is pointwise proper at $\omega$ we have the following:

(1) The notions of valuation equilibrium, Walrasian valuation equilibrium, and weak Pareto optimality coincide.

(2) If the initial endowments are decomposable, then the notions of equilibrium, Walrasian equilibrium, and Edgeworth equilibrium coincide.

(3) If the economy is compact and the initial endowments are decomposable, then there exists a Walrasian equilibrium.

\section{The indispensability of proper price spaces}

We have seen that the properness of prices provides sufficient conditions for the decentralization of optimal allocations by linear prices. In this section we show that basically the properness of the non-linear prices also affords necessary conditions for linear decentralization. That is, one cannot hope to derive the classical Walrasian theory of value for proper economies unless the prices have some properness properties at the total endowment vector. We begin with a definition.

Definition 25 For a price of the form $p=\bigvee_{i=1}^{m} f_{i}$ with $f_{i} \in L_{+}^{\prime}$ for each $i$, we say that an allocation $x \in A_{\omega}$ is an optimizer if $p(\omega)=\sum_{i=1}^{m} f_{i}\left(x_{i}\right)$. The allocation $x$ is a non-trivial optimizer if $p(\omega)>0$ and $x_{i}>0$ for all $i$.

We are ready to establish the equivalence between pointwise proper price spaces and the decentralization of Edgeworth equilibria by linear prices.

Theorem 26 For the collection $\mathcal{E}_{D}(L, \omega)$ of decomposable economies the following statements are equivalent:

(a) Each price of the form $p=\bigvee_{i=1}^{m} f_{i}$ that has a non-trivial optimizer is pointwise proper at $\omega$. 
(b) Every Edgeworth equilibrium is a Walrasian equilibrium.

proof.

$(a) \Longrightarrow(b)$ Let $x=\left(x_{1}, \ldots, x_{m}\right)$ be an Edgeworth equilibrium for some economy in $\mathcal{E}_{D}(L, \omega)$. Clearly, $x$ is a non-trivial allocation. According to Theorem 16 , the allocation $x$ is an equilibrium for some price of the form $p=\bigvee_{i=1}^{m} f_{i}$ with $p(\omega)>0$ for which $x$ is an optimizer.

Now, by our assumption, $p$ is pointwise proper at $\omega$ and a glance at part (3) of Lemma 21 guarantees that $x$ is a Walrasian equilibrium.

$(b) \Longrightarrow(a)$ Let a price of the form $p=\bigvee_{i=1}^{m} f_{i}$ with $p(\omega)>0$ have a non-trivial optimizer $x=\left(x_{1}, \ldots, x_{m}\right)$. That is, $x_{i}>0$ for all $i$ and $p(\omega)=\sum_{i=1}^{m} f_{i}\left(x_{i}\right)$.

Fix a strictly positive linear functional $h$ on $L$ and put $g_{i}=f_{i}+h$ for each $i$ and consider the price $q=\bigvee_{i=1}^{m} g_{i}=p+h$. We claim that $q$ is pointwise proper at $\omega$.

Clearly, $x=\left(x_{1}, \ldots, x_{m}\right)$ is an optimizer for $q$. That is, $q(\omega)=\sum_{i=1}^{m} g_{i}\left(x_{i}\right)>0$. From $q\left(x_{i}\right) \geq g_{i}\left(x_{i}\right)$ and the inequalities

$$
q(\omega)=q\left(\sum_{i=1}^{m} x_{i}\right) \geq \sum_{i=1}^{m} q\left(x_{i}\right) \geq \sum_{i=1}^{m} g_{i}\left(x_{i}\right)=q(\omega),
$$

it follows that $g_{i}\left(x_{i}\right)=q\left(x_{i}\right)$ holds for all $i$.

Next, we shall define an economy in $\mathcal{E}_{D}(L, \omega)$ for which $x$ is an Edgeworth equilibrium. To this end, for each $i$ let $\omega_{i}=\frac{g_{i}\left(x_{i}\right)}{q(\omega)} \omega>0$ and note that $q\left(\omega_{i}\right)=g_{i}\left(x_{i}\right)$ holds for each $i$. Furthermore, for each trader $i=1, \ldots, m$ let $g_{i}$ be the trader's utility function. Clearly, this economy is in $\mathcal{E}_{D}(L, \omega)$.

Since $x \in L_{+}$with $g_{i}(x)>g_{i}\left(x_{i}\right)$ implies $q(x) \geq g_{i}(x)>g_{i}\left(x_{i}\right)=q\left(x_{i}\right)$, it follows that $q$ supports $x$ as a valuation equilibrium. We need to show that $q$ supports $x$ as an equilibrium. To this end, let $\alpha_{1}, \ldots, \alpha_{m}$ be arbitrary non-negative scalars. Then we have

$$
\sum_{i=1}^{m} \alpha_{i} q\left(x_{i}\right)=\sum_{i=1}^{m} \alpha_{i} g_{i}\left(x_{i}\right)=\sum_{i=1}^{m} \alpha_{i} q\left(\omega_{i}\right)=q\left(\sum_{i=1}^{m} \alpha_{i} \omega_{i}\right)
$$

which tells us that $x$ is an equilibrium and thus an Edgeworth equilibrium. 
Therefore, by assumption $x$ is a Walrasian equilibrium for some non-zero price $\pi \in$ $L_{+}^{\prime}$, which is clearly strictly positive. We can assume without loss of generality that $\pi(\omega)=q(\omega)$. Clearly, $\pi\left(x_{i}\right)=\pi\left(\omega_{i}\right)=g_{i}\left(x_{i}\right)$. By Lemma 12 it must be the case that $\pi \geq g_{i}$ for each $i$ and thus $\pi \geq q$.

Finally, notice that from the inequality $\pi \geq q=p+h$ we get $\pi-h \geq p>0$. Moreover, from $(\pi-h)(\omega)=(q-h)(\omega)=p(\omega)>0$ and the fact that if $y \in L_{+}$ satisfies $p(y)>p(\omega)$, then $(\pi-h)(y) \geq q(y)-h(y)=p(y)>p(\omega)=(\pi-h)(\omega)$, it follows that $\pi-h$ supports the set $\left\{y \in L_{+}: p(y)>p(\omega\}\right.$ at $\omega$. Therefore, by part (1) of Lemma 19, we see that $p$ is pointwise proper at $\omega$.

Let us move on to the second welfare theorem. We begin by introducing one more notion of pointwise properness for prices.

Definition 27 We say that a price of the form $p=\bigvee_{i=1}^{m} f_{i}$ is $\omega$-scalable at $x \in \mathcal{A}_{\omega}$ if there exist multipliers $\lambda_{1}, \ldots, \lambda_{m}$ satisfying $0<\lambda_{i} \leq 1$ for each $i$ such that:

(1) the allocation $x$ is an optimizer for the new price $q=\bigvee_{i=1}^{m} \lambda_{i} f_{i}$, and

(2) the price $q$ is pointwise proper at $\omega$.

Now we can state and prove the main result of this section. It presents necessary and sufficient conditions for the Walrasian decentralization of optimal allocations.

Theorem 28 In the set of proper economies $\mathcal{E}(L, \omega)$ the following statements are equivalent:

(a) Every price of the form $p=\bigvee_{i=1}^{m} f_{i}$ with each $f_{i}$ strictly positive is $\omega$-scalable at each of its non-trivial optimizers.

(b) Every non-trivial Pareto optimal allocation is a Walrasian valuation equilibrium.

proof. $(\mathrm{a}) \Longrightarrow(\mathrm{b})$ Let $x=\left(x_{1}, \ldots, x_{m}\right)$ be a non-trivial Pareto optimal allocation for some economy in $\mathcal{E}(L, \omega)$. From parts (1) and (3) of Theorem 16 this allocation is supported as a valuation equilibrium by a price $p=\bigvee_{i=1}^{m} f_{i}$ for which $x$ is an optimizer. So, we have $p(\omega)=\sum_{i=1}^{m} p_{i}\left(x_{i}\right)=\sum_{i=1}^{m} f_{i}\left(x_{i}\right)$. Since $p\left(x_{i}\right) \geq f_{i}\left(x_{i}\right)$ for each $i$, it follows that $p\left(x_{i}\right)=f_{i}\left(x_{i}\right)$ for each $i$. Furthermore, the proof of $[13$, 
Theorem 5.1(1)] shows that the functions $f_{i}$ can be chosen in such a way that each $f_{i}$ (strictly) supports $P_{i}\left(x_{i}\right)$ at $x_{i}$, i.e., $y \in P_{i}\left(x_{i}\right)$ implies $f_{i}(y)>f_{i}\left(x_{i}\right)$.

We need to show that each $f_{i}$ is strictly positive. From $p(\omega)=\sum_{i=1}^{m} f_{i}\left(x_{i}\right)>0$ it must be the case that $f_{j}\left(x_{j}\right)>0$ for some $j$. Notice that if $z \in P_{j}\left(x_{j}\right)$ and $f_{j}(z)=f_{j}\left(x_{j}\right)$, then $f_{j}(\alpha z)<f_{j}\left(x_{j}\right)$ for all $\alpha<1$; which is impossible since $P_{j}$ has open values in some linear topology on $L$. Therefore, for $z>0$, it must be the case that $f_{j}\left(x_{j}+z\right)>f_{j}\left(x_{j}\right)$, which implies that $f_{j}(z)>0$. This in turn implies that $f_{j}$ and $p$ are strictly positive, which further implies that $f_{i}\left(x_{i}\right)=p\left(x_{i}\right)>0$ for each $i$. By the same argument we see that each $f_{i}$ is strictly positive.

Now by our assumption there exist scalars $\lambda_{1}, \ldots, \lambda_{m}$ with $0<\lambda_{i} \leq 1$ for each $i$ such that $x$ remains an optimizer of the price $q=\bigvee_{i=1}^{m} \lambda_{i} f_{i}$ that is pointwise proper at the point $\omega$.

Next, we shall show that $q$ also supports the allocation $x$ as a valuation equilibrium. Clearly, $q\left(x_{i}\right) \geq \lambda_{i} f_{i}\left(x_{i}\right)$ for each $i$ and since $x$ is an optimizer for $q$ we have

$$
q(\omega)=\sum_{i=1}^{m} \lambda_{i} f_{i}\left(x_{i}\right) \leq \sum_{i=1}^{m} q\left(x_{i}\right) \leq q(\omega) .
$$

Consequently, for each $i$ we have $q\left(x_{i}\right)=\lambda_{i} f_{i}\left(x_{i}\right)$ and $\sum_{i=1}^{m} q\left(x_{i}\right)=q(\omega)>0$. Now if $z \in P_{i}\left(x_{i}\right)$, then $q(z) \geq \lambda_{i} f_{i}(z)>\lambda_{i} f_{i}\left(x_{i}\right)=q\left(x_{i}\right)$, and this shows that $q$ supports $x$ as a valuation equilibrium. Now the validity of (b) follows from part (1) of Lemma 21.

(b) $\Longrightarrow(\mathrm{a})$ Assume that (b) is true and let an allocation $x=\left(x_{1}, \ldots, x_{m}\right)$ be a non-trivial optimizer for a price $p=\bigvee_{i=1}^{m} f_{i}$ with each $f_{i}$ strictly positive.

Next, we consider an economy with $m$ consumers, total endowment $\omega$, and utility functions given by the strictly positive linear functionals $f_{i}$. Thus, the preference correspondence $P_{i}: L_{+} \rightarrow L_{+}$of consumer $i$ is defined by $P_{i}(y)=\left\{x \in L_{+}: f_{i}(x)>\right.$ $\left.f_{i}(y)\right\}$. Clearly, this economy is $\omega$-proper and since $x$ is an optimizer it is Pareto optimal. So, according to our hypothesis, there exists a non-zero price $\pi \in L_{+}^{\prime}$ that supports $x$ as a valuation equilibrium. That is, if $z \in L_{+}$satisfies $f_{i}(z)>f_{i}\left(x_{i}\right)$, then $\pi(z)>\pi\left(x_{i}\right)$. By the strict positivity of $f_{i}$ we see that $f_{i}\left(2 x_{i}\right)>f_{i}\left(x_{i}\right)$ and so $2 \pi\left(x_{i}\right)>\pi\left(x_{i}\right)$. Consequently, $\pi\left(x_{i}\right)>0$ holds for each $i$. Without loss of generality we can assume that $0<\pi\left(x_{i}\right)<f_{i}\left(x_{i}\right)$ also holds for each $i$. 
Now for each $i$ let $\lambda_{i}=\frac{\pi\left(x_{i}\right)}{f_{i}\left(x_{i}\right)}$ and note that $0<\lambda_{i}<1$. For each $i$ consider the strictly positive linear functional $g_{i}=\lambda_{i} f_{i}$. Observe that $g_{i}\left(x_{i}\right)=\pi\left(x_{i}\right)$ for each $i$. Moreover, if some vector $z \in L_{+}$satisfies $g_{i}(z)=g_{i}\left(x_{i}\right)>0$, or $f_{i}(z)=f_{i}\left(x_{i}\right)$, then $f_{i}(\delta z)>f_{i}\left(x_{i}\right)$ for all $\delta>1$ and so $\delta \pi(z)>\pi\left(x_{i}\right)$ for all $\delta>1$, from which it follows that $\pi(z) \geq \pi\left(x_{i}\right)=g_{i}\left(x_{i}\right)$. Now a glance at Lemma 12 guarantees that $\pi \geq g_{i}$ for each $i$. This implies $\pi \geq \bigvee_{i=1}^{m} g_{i}$, and so from

$$
\pi(\omega) \geq\left[\bigvee_{i=1}^{m} g_{i}\right](\omega) \geq \sum_{i=1}^{m} g_{i}\left(x_{i}\right)=\sum_{i=1}^{m} \pi\left(x_{i}\right)=\pi(\omega),
$$

we see that $\left(x_{1}, \ldots, x_{m}\right)$ is an optimizer for $\bigvee_{i=1}^{m} g_{i}$ and that $\pi(\omega)=\left[\bigvee_{i=1}^{m} g_{i}\right](\omega)>0$.

Now if an arbitrary vector $z \in L_{+}$satisfies $\left[\bigvee_{i=1}^{m} g_{i}\right](z)>\left[\bigvee_{i=1}^{m} g_{i}\right](\omega)$, then we have the inequalities $\pi(z) \geq\left[\bigvee_{i=1}^{m} g_{i}\right](z)>\left[\bigvee_{i=1}^{m} g_{i}\right](\omega)=\pi(\omega)$. This shows that $\pi$ supports the set $\left\{z \in L_{+}:\left[\bigvee_{i=1}^{m} g_{i}\right](z)>\left[\bigvee_{i=1}^{m} g_{i}\right](\omega)\right\}$ at $\omega$ and from this and part (a) of Lemma 19, we see that the price $\bigvee_{i=1}^{m} g_{i}$ is pointwise proper at $\omega$.

We conclude this section by showing that the existence of an equilibrium implies that prices are $\omega$-scalable at each of their non-trivial optimizers.

Theorem 29 If for each economy in $\mathcal{E}(L, \omega)$ there exists a Walrasian equilibrium, then every price $p=\bigvee_{i=1}^{m} f_{i}$ with each $f_{i}$ strictly positive is $\omega$-scalable at each of its non-trivial optimizers.

proof. Let an allocation $x=\left(x_{1}, \ldots, x_{m}\right)$ be a non-trivial optimizer for a price $p=\bigvee_{i=1}^{m} f_{i}$ with each $f_{i}$ strictly positive. Consider an economy with $m$ consumers, initial endowments $\omega_{i}=x_{i}$ for each $i$, and utility functions given by the strictly positive linear functionals $f_{i}$. Clearly, this economy is $\omega$-proper.

According to our hypothesis, there exists a Walrasian equilibrium $y=\left(y_{1}, \ldots, y_{m}\right)$ for this economy supported by non-zero price $\pi \in L_{+}^{\prime}$. Since $y$ is individually rational and since $x$ is an optimizer for the price $p$ and $f_{i}\left(y_{i}\right) \geq f_{i}\left(\omega_{i}\right)=f_{i}\left(x_{i}\right)$ holds for each $i$ it must be the case that $f_{i}\left(y_{i}\right)=f_{i}\left(x_{i}\right)$ for each $i$. Since $\pi\left(y_{i}\right)=\pi\left(\omega_{i}\right)=\pi\left(x_{i}\right)$ for each $i$, we see that if $z \in L_{+}$satisfies $f_{i}(z)>f_{i}\left(x_{i}\right)$, then $\pi(z)>\pi\left(x_{i}\right)$. It is now easy to follow the proof of Theorem 28 and show that $p$ is $\omega$-scalable at $x$. 


\section{Properness of prices in financial models}

The previous theorems demonstrate that we need some pointwise properness of prices if we are to derive the Walrasian value theory. We start with an example that guarantees the $\omega$-properness of prices in $\mathcal{P}$.

Recall that in our model of portfolio trading we have two spaces linked by the portfolio returns operator as follows:

(1) The portfolio space $L$ is ordered by the portfolio dominance ordering.

(2) The space of contingent claims $X$ is a Banach lattice.

(3) The portfolio returns operator $R: L \hookrightarrow X$ is one-to-one and positive and pulls back order intervals of the marketed space $\mathcal{M}$ to closed and bounded dominance intervals of $L$.

Here is an example of our finance model with an $\omega$-proper price space.

Example 1 Let $L=\ell_{2}$ and $X=L_{2}[0,1]$ and consider the operator $R: L \rightarrow X$ defined on the basic vectors $e_{1}, e_{2}, \ldots$ of $\ell_{2}$ by

(1) $R\left(e_{1}\right)=\chi_{\left[\frac{1}{2}, 1\right]}+\chi_{\left[\frac{1}{5}, \frac{1}{3}\right]}$,

(2) $R\left(e_{2}\right)=\chi_{\left[\frac{1}{3}, 1\right]}$,

(3) $R\left(e_{3}\right)=\chi_{\left[\frac{1}{4}, \frac{1}{2}\right]}$, and

(4) $R\left(e_{n}\right)=\lambda_{n} \chi_{\left[\frac{1}{n+2}, \frac{1}{n+1}\right]}$ for each $n \geq 4$, where the scalars $\lambda_{n}$ satisfy $\lambda_{n}>0$ and they are appropriately chosen so that $R$ is indeed an operator from $\ell_{2}$ to $L_{2}[0,1]$.

As usual, if $\left(\alpha_{1}, \alpha_{2}, \ldots\right) \in \ell_{2}$, then we have

$$
R\left(\alpha_{1}, \alpha_{2}, \ldots\right)=\sum_{n=1}^{\infty} \alpha_{n} R\left(e_{n}\right)
$$

Clearly, the operator $R$ is one-to-one and positive (and hence continuous).

It should not be difficult to see that if $\lambda_{n}=1$ for each $n \geq 4$, then the operator $R$ is not bounded below. On the other hand if $\lambda_{n}=\sqrt{(n+1)(n+2)}$ for each $n \geq 4$, then the operator $R$ is bounded below. In fact, it is easy to verify that when $\lambda_{n}=1$ for each $n \geq 4$ the operator $R$ satisfies the following properties: 
(a) As in every model, the portfolio dominance cone on $\ell_{2}$ is closed and generating. Moreover, $R$ pulls back order intervals of $\mathcal{M}$ to closed and bounded dominance intervals in $L$.

(b) The portfolio dominance ordering on $L$ is not a lattice ordering.

(c) The price space $\mathcal{P}$ is $\omega$-proper for every positive portfolio $\omega \in L_{+}$.

Therefore our results on linear decentralization apply to this example.

Let $\mathcal{M}^{\prime}$ be the dual space of the marketed space $\mathcal{M}$ and assume that $R$ is bounded below. Then considering $R: L \rightarrow \mathcal{M}$ as an order isomorphism, we see that its adjoint $R^{\prime}: \mathcal{M}^{\prime} \rightarrow L^{\prime}$, defined via the duality identity

$$
\left\langle R^{\prime}(f), m\right\rangle=\langle f, R(m)\rangle=f(R(m))
$$

for all $m \in \mathcal{M}$ and all $f \in \mathcal{M}^{\prime}$, is also an order isomorphism. This operator associates each state dependent price system with a securities price. We call this operator the securities prices operator.

We conclude the paper with the following very useful characterization of pointwise proper prices in terms of a solution to a linear minimization problem. Its proof follows immediately from Lemma 20.

Theorem 30 Assume that order intervals of $L$ are weakly compact and that the payoff operator $R$ is bounded from below. Let $f_{1}, \ldots, f_{m} \in L_{+}^{\prime}$ and $\omega>0$ be such that the price $p=\bigvee_{i=1}^{m} f_{i}$ satisfies $p(\omega)>0$. Then the following three conditions are equivalent:

(1) The price $p$ is pointwise proper at $\omega$.

(2) There exists a solution to the following linear minimization problem:

$$
\min g(R(\omega)) \quad \text { subject to: } \quad g \in \mathcal{M}^{\prime} \text { and } R^{\prime}(g) \geq f_{i} \text { for all } i=1, \ldots, m \text {. }
$$

(3) There exists a solution to the following linear minimization problem:

$$
\min h(R(\omega)) \quad \text { subject to: } \quad h \in X^{\prime} \text { and } R^{\prime}(h) \geq f_{i} \text { for all } i=1, \ldots, m \text {, }
$$

where now $R^{\prime}$ denotes the adjoint of the operator $R: L \rightarrow X$. 
Ackowledgements. The paper has benefited from the comments of an anonymous referee. The paper was prepared during visits of Aliprantis to CERMSEM, of Aliprantis and Florenzano to the Economic Theory Centre of the University of Melbourne, and of Florenzano and Tourky to Purdue University. The hospitality of these institutions is gratefully acknowledged. The research of Aliprantis is supported by the NSF Grants EIA0075506, SES-0128039 and DMI-0122214 and the DOD Grant ACI-0325846. The research of R. Tourky is funded by the Australian Research Council Grant A00103450.

\section{References}

[1] Y. A. Abramovich and C. D. Aliprantis. An Invitation to Operator Theory, Vol. 50, Graduate Studies in Mathematics, Amer. Math. Soc., Providence, RI, 2002.

[2] C. D. Aliprantis and K. C. Border, Infinite Dimensional Analysis: A Hitchhiker's Guide, 2nd. ed., Springer-Verlag, Heidelberg and New York, 1999.

[3] C. D. Aliprantis, D. J. Brown, and O. Burkinshaw, Edgeworth equilibria, Econometrica 55 (1987), 1109-1137.

[4] C. D. Aliprantis, D. J. Brown, and O. Burkinshaw, Edgeworth equilibria in production economies, J. Econ. Theory 43 (1987), 252-291.

[5] C. D. Aliprantis, D. J. Brown, I. A. Polyrakis, and J. Werner, Portfolio dominance and optimality in infinite security markets, J. Math. Econ. 30 (1998), 347-366.

[6] C. D. Aliprantis and O. Burkinshaw, Locally Solid Riesz Spaces, Academic Press, New York and London, 1978.

[7] C. D. Aliprantis and O. Burkinshaw, Positive Operators, Academic Press, New York and London, 1985.

[8] C. D. Aliprantis and O. Burkinshaw, When is the core equivalence theorem valid?, Econ. Theory 1 (1991), 169-182.

[9] C. D. Aliprantis, P. K. Monteiro, and R. Tourky, Non-marketed options, non-existence of equilibria, and non-linear prices, J. Econ. Theory 114 (2004), 345-357.

[10] C. D. Aliprantis and R. Tourky, The super order dual of an ordered vector space and the Riesz-Kantorovich formula, Trans. Amer. Math. Soc. 354 (2002), 2055-2077.

[11] C. D. Aliprantis and R. Tourky, Markets that don't replicate any option, Econ. Letters 76 (2002), 437-442. 
[12] C. D. Aliprantis, R. Tourky, and N. C. Yannelis, Cone conditions in general equilibrium theory, J. Econ. Theory 92 (2000), 96-121.

[13] C. D. Aliprantis, R. Tourky, and N. C. Yannelis, A theory of value: equilibrium analysis beyond vector lattices, J. Econ. Theory 100 (2001), 22-72.

[14] T. Andô, On fundamental properties of a Banach space with a cone, Pacific J. Math. 12 (1962), 1163-1169.

[15] A. Araujo and P. K. Monteiro, Equilibrium without uniform conditions, J. Econ. Theory 48 (1989), 416-427.

[16] K. Back, Structure of consumption sets and existence of equilibria in infinitedimensional spaces, J. Math. Econ. 17 (1988), 89-99.

[17] S. A. Clark, A tangent cone analysis of smooth preferences on a topological vector space, Econ. Theory 23 (2004), 337-352.

[18] G. Debreu, New concepts and techniques for equilibrium analysis, Int. Econ. Rev. 3 (1962), 257-273.

[19] G. Debreu and H. Scarf, A limit theorem on the core of an economy, Int. Econ. Rev. 4 (1963), 235-246.

[20] M. Deghdak and M. Florenzano, Decentralizing Edgeworth equilibria in economies with many commodities, Econ. Theory 14 (1999), 297-310.

[21] M. Florenzano, Edgeworth equilibria, fuzzy core and equilibria of a production economy without ordered preferences, J. Math. Anal. Appl. 153 (1990), 18-36.

[22] M. Florenzano and V. Marakulin, Production equilibria in vector lattices, Econ. Theory $\mathbf{1 7}$ (2001), 577-598.

[23] V. Galvani, Financial Markets and the Theory of Riesz Spaces, Ph.D. Dissertation, Università degli Studi di Brescia, Italy, January 2002.

[24] R. A. Jarrow, X. Jin, and D. B. Madan, The second fundamental theorem of asset pricing, Math. Finance 9 (2001), 255-273.

[25] L. E. Jones, Special problems arising in the study of economies with infinitely many commodities, in: H. F. Sonnenschein, ed., Models of Economic Dynamics, Lecture Notes in Economics and Mathematical Systems, \# 264, Springer-Verlag, New York and Berlin, 184-205, 1986.

[26] I. Karatzas and S. E. Shreve, Methods of Mathematical Finance, Springer-Verlag, Heidelberg and New York, 1998. 
[27] W. A. J. Luxemburg and A. C. Zaanen, Riesz spaces, Vol. I, North-Holland Publishing Co., Amsterdam, 1971.

[28] M. Magill and M. Quinzii, Theory of Incomplete Markets, MIT Press, Cambridge and London, 1996.

[29] A. Mas-Colell, The price equilibrium existence problem in topological vector lattices, Econometrica 54 (1986), 1039-1055.

[30] A. Mas-Colell and S. F. Richard, A new approach to the existence of equilibria in vector lattices, J. Econ. Theory 53 (1991), 1-11.

[31] A. L. Peressini, Ordered Topological Vector Spaces, Harper and Row, New York and London, 1967.

[32] K. Podczeck, Equilibria in vector lattices without ordered preferences or uniform properness, J. Math. Econ. 25 (1996), 465-485.

[33] S. F. Richard, A new approach to production equilibria in vector lattices, J. Math. Econ. 18 (1989), 41-56.

[34] S. F. Richard and W. R. Zame Proper preferences and quasiconcave utility functions. J. Math. Econ. 15 (1986), 231-247.

[35] S. Ross, Options and efficiency, Quart. J. Econ. 90 (1976), 75-89.

[36] H. H. Schaefer, Topological Vector Spaces, Springer-Verlag, New York and Berlin, 1971.

[37] R. Tourky, A new approach to the limit theorem on the core of an economy in vector lattices, J. Econ. Theory 78 (1998), 321-328.

[38] R. Tourky, The limit theorem on the core of a production economy in vector lattices with unordered preferences, Econ. Theory 14(1999), 219-226.

[39] C. Le Van, Complete characterization of Yannelis-Zame and Chichilnisky-KalmanMas-Colell properness for separable concave functions defined in $L_{+}^{p}$ and $L^{p}$, Econ. Theory 8 (1996), 155-165.

[40] N. C. Yannelis and W. R. Zame, Equilibria in Banach lattices without ordered preferences, J. Math. Econ. 15 (1986), 85-110.

[41] W. R. Zame, Competitive equilibria in production economies with an infinitedimensional space, Econometrica 55 (1987), 1075-1108. 